\title{
Changing Productivity, Variability, and Synchrony within Stock Aggregates can Limit Management Effectiveness
}

\author{
Cameron Freshwater ${ }^{1}$, Sean C. Anderson ${ }^{1}$, Kendra R. Holt ${ }^{2}$, Ann-Marie Huang ${ }^{1}$, and Carrie A. Holt ${ }^{1}$ \\ ${ }^{\text {I}}$ Fisheries and Oceans Canada, Pacific Biological Station, 3190 Hammond Bay Road, Nanaimo, BC, Canada \\ ${ }^{2}$ Fisheries and Oceans Canada, Institute of Ocean Sciences, 9860 West Saanich Road, Sidney, BC, Canada
}

Keywords: population dynamics, portfolio effects, simulation model

Asynchrony among component populations can increase the temporal stability of ecological aggregates, a process commonly referred to as the portfolio effect (Doak et al. 1998; Tilman 1999). Within salmon species, population diversity reduces aggregate variability in spawner returns and catches, as well as the probability of fishery closures (Schindler et al. 2010) and can increase long-term resilience given environmental uncertainty (Anderson et al. 2015). Aggregate variability is linked to two metrics - the weighted mean coefficient of variation among components (component variability) and an index of synchrony (Loreau and de Mazancourt 2008; Thibaut and Connolly 2013). Component variability and synchrony may increase due to anthropogenic disturbance (Griffiths et al. 2014) or large-scale environmental processes (Kilduff et al. 2015), resulting in less predictable returns, boom-and-bust fisheries, and increased risk of overharvest. Greater aggregate variability may also be exacerbated by declines in population productivity associated with reduced survival or fecundity (Peterman and Dorner 2012; Minto et al. 2014; Britten et al. 2016). Although changes in component variability, synchrony, and productivity may independently constrain fisheries management, it is not necessarily clear how these processes will interact. We paired a retrospective analysis of observed trends in the dynamics of Fraser River sockeye salmon $(O$. nerka) with a closed-loop simulation model to evaluate the impact of greater aggregate variability under various productivity regimes (Freshwater et al. in press). The closed-loop simulation is analogous to the quantitative component of a management strategy evaluation (MSE; Punt et al. 2016) and includes a biological model, as well as a model of the mixed-stock fishery that harvests these populations.

The retrospective analysis indicated that generational-means (i.e., 4-year) of Fraser River sockeye salmon productivity declined from the late 1980s through 2005 , stabilized for several years, then began to decline again (Fig. 1a). Component variability in recruit abundance exhibited interdecadal cycles, increasing by approximately $50 \%$ in the 1950s, 1980s, and 2010s, then declining (Fig. 1b). Similarly, synchrony was high in the 1950s, low and stable through the 1980s, and increased rapidly beginning in 1990 (Fig. 1c).
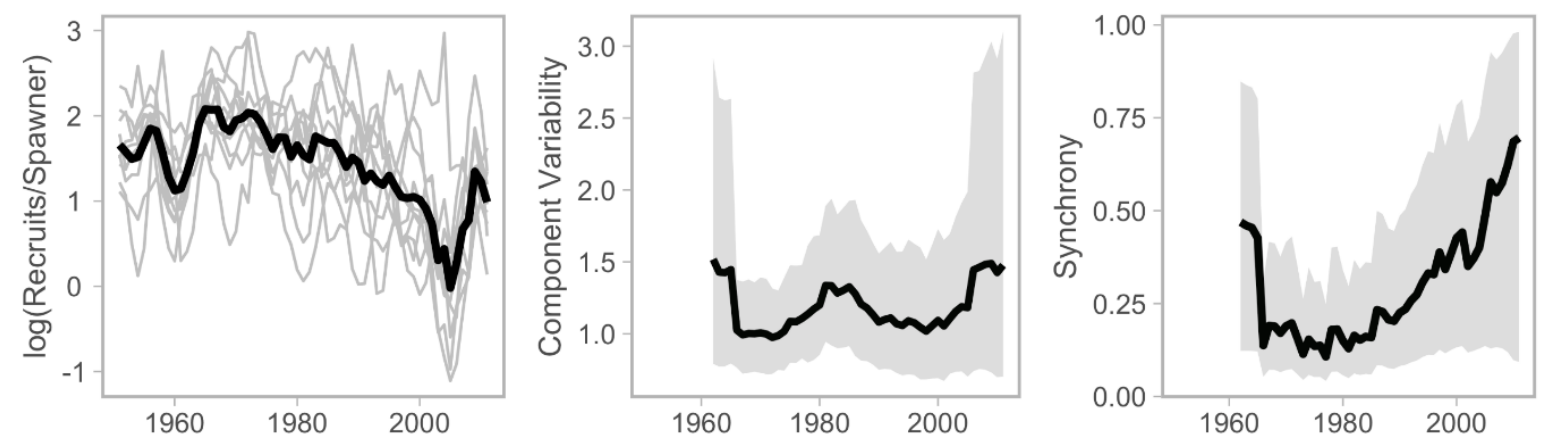

Fig. 1. Observed trends in stock-specific log (recruits per spawner) (a), mean component coefficient of variation (b), and synchrony index (c). All values are lagged by four years (one generation; productivity) or 12 years (three generations; component variability and synchrony). In panel (a) grey lines represent productivity trends for 10 individual stocks and the mean (black line). In panels (b) and (c), black lines represent estimates and grey bands represent $95 \%$ confidence intervals.

Simulations demonstrated that the effect of increasing component variability and synchrony differed among performance metrics and was moderated by the underlying productivity regime. When productivity was simulated at its reference value, greater synchrony led to declines in median aggregate return abundance ( 7-16\% across component variability scenarios; different colour symbols in Fig. 2a). Conversely, aggregate return abundance

All correspondence should be addressed to C. Freshwater. 
increased by $\sim 9 \%$ when component variability was high and synchrony was low (purple symbols Fig. 2a) due to individual stocks experiencing more frequent large positive recruitment deviations. Since high component variability increases the skew of the log-normal distribution of stock-specific recruitment, the normalizing effects of asynchronous dynamics are more evident when component variability is high (Fig. 2a). Increased synchrony also reduced the proportion of management units (groups of stocks harvested in the same marine fishery; MUs) above their escapement goal (Fig. 2c). These declines are driven by reduced aggregate abundance when synchrony is high (Fig. 2a), as well as declines in performance in stock-specific abundance as component variability increases. Finally, increasing variability and synchrony simultaneously lead to severe declines in aggregate catch stability ( 40\%; Fig. 2e).
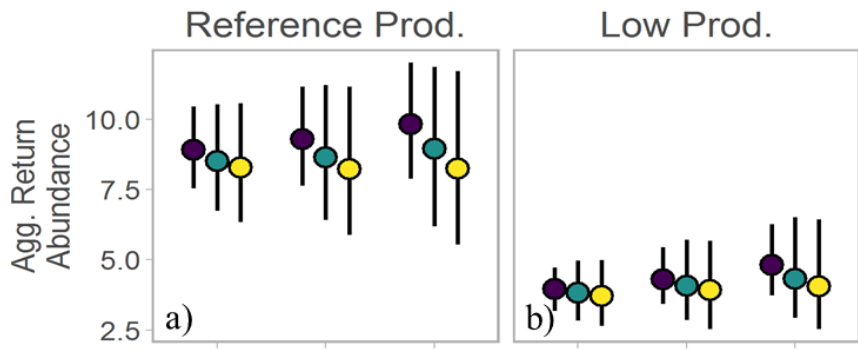

Fig. 2. Effects of component variability and synchrony for reference and low productivity scenarios on three performance metrics: aggregate return abundance $(a, b)$, proportion of years escapement goals met (c, d), and catch stability (e, f). Points represent medians and whiskers $90 \%$ probability intervals among 1500 Monte Carlo trials.
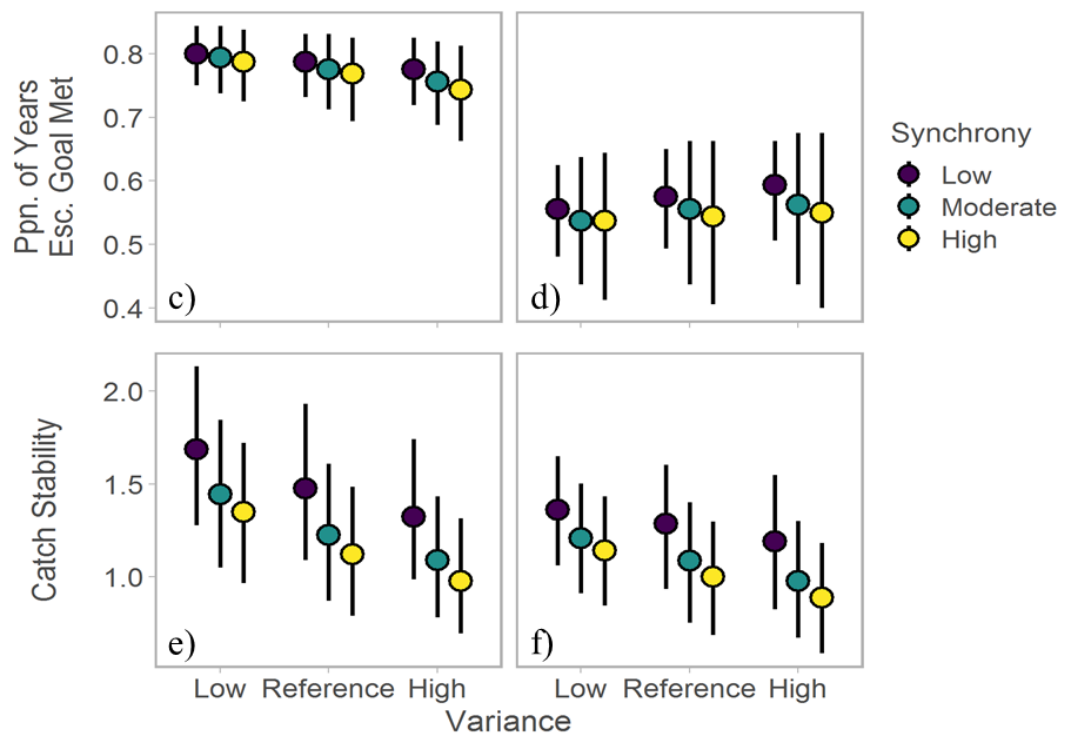

Median performance declined under the low productivity scenario for all performance metrics (Fig. 2b, d, f). Relative to the reference productivity scenario, variation among trials in return abundance declined (Fig. 2b), while variation in the proportion of MUs above their escapement goals increased (Fig. 2d). Greater component variability also increased the probability that these escapement goals would be exceeded (i.e. the effect of component variance was reversed relative to reference scenario; Fig. 2d). Finally, the effects of greater synchrony on catch stability were reduced when productivity was low (Fig. 2f).

Pacific salmon populations often exhibit evidence of reduced productivity (Peterman and Dorner 2012; Dorner et al. 2018), increased variability (Satterthwaite and Carlson 2015), and increased synchrony (Kilduff et al. 2015; Satterthwaite and Carlson 2015; Freshwater et al. 2018); however, the cumulative impacts of each process are rarely considered simultaneously. Here we show that increases in component variability and synchrony will have severe impacts on catch stability, but relatively modest effects on aggregate return abundance. Such patterns are concerning because they suggest harvests in weakened ecological portfolios will increasingly be driven by boomand-bust cycles even if median catches remain high over longer time horizons. Greater variability in catches may have strong negative impacts on communities that rely on stable fishing opportunities. Indeed, observed declines in aggregate stability have been associated with substantial socio-economic costs, as documented by the federal inquiry into declines in Fraser River sockeye salmon (Cohen 2012; Peterman and Dorner 2012). Additionally, we show that most performance metrics were more strongly impacted by reductions in productivity than component variability or 
synchrony, but that the interactions among these processes are not uniform. In some cases, declines in productivity reduced or even reversed the negative impacts of greater component variability and synchrony.

These simulations have several implications for managers responsible for multistock fisheries or other ecological aggregates. First, we found that declines in productivity and increases in aggregate variability will have multiplicative effects that may constrain management objectives. The magnitude of these effects will be determined by whether changes in aggregate variability are driven by component variability or synchrony, how conservationvs. catch-based objectives are prioritized, and whether the focal unit of concern are individual stocks, the aggregate as a whole, or an intermediate unit. Second, even relatively conservative, abundance-based harvest control rules (such as the one included in our simulation) appear to be sensitive to changes in component variability, synchrony, and productivity. Less responsive or data limited harvest control rules may be even more vulnerable to weakening portfolio effects.

Systems-based approaches are increasingly favored in natural resource disciplines such as fisheries because they can stabilize the availability of ecosystem services (Link 2018). Climate change and other anthropogenic impacts are likely to increase component variability and synchrony, while reducing productivity, which is likely to weaken portfolio effects and reduce the efficacy of systems-based approaches. Since stability can be increased along multiple axes of biodiversity the most precautionary approach is to main intact ecological portfolios wherever possible.

\section{REFERENCES}

Anderson, S.C., J.W. Moore, M.M. McClure, N.K. Dulvy, and A.B. Cooper. 2015. Portfolio conservation of metapopulations under climate change. Ecol. Appl. 25: 559-572.

Britten, G.L., M. Dowd, and B. Worm. 2016. Changing recruitment capacity in global fish stocks. Proc. Nat. Acad. Sci. USA 113: 134-139.

Carlson, S.M., and W.H. Satterthwaite. 2011. Weakened portfolio effect in a collapsed salmon population complex. Can. J. Fish. Aquat. Sci. 68: 1579-1589.

Cohen, B.I. 2012. The Uncertain Future of Fraser River Sockeye - Part 1. Cohen Commission. 692 pp.

Doak, D.F., D. Bigger, E.K. Harding, M.A. Marvier, R.E.O. Malley, and D. Thomson. 1998. The statistical inevitability of stability-diversity relationships in community ecology. Am. Nat. 151:264-276.

Dorner, B., M.J. Catalano, and R.M. Peterman. 2018. Spatial and temporal patterns of covariation in productivity of Chinook salmon populations of the northeastern Pacific Ocean. Can. J. Fish. Aquat. Sci. 75:1082-1095.

Freshwater, C., B.J. Burke, M.D. Scheuerell, S.C.H. Grant, M. Trudel, and F. Juanes. 2018. Coherent population dynamics associated with sockeye salmon juvenile life history strategies. Can. J. Fish. Aquat. Sci. 75:13461356.

Freshwater, C., S.C. Anderson, K.R. Holt, A.-M. Huang, and C.A. Holt. In press. Weakened portfolio effects constrain maangement effectiveness for population aggregates. Ecol. Appl.

Griffiths, J.R., D.E. Schindler, J.B. Armstrong, M.D. Scheuerell, D.C. Whited, R.A. Clark, R. Hilborn, C.A. Holt, S.T. Lindley, J.A. Stanford, and E.C. Volk. 2014. Performance of salmon fishery portfolios across western North America. J. Appl. Ecol. 51:1554-1563.

Kilduff, D.P., E. Di Lorenzo, L.W. Botsford, and S.L. Teo. 2015. Changing central Pacific El Ninos reduce stability of North American salmon survival rates. Proc. Nat. Acad. Sci. USA 112: 10962-10966.

Link, J. 2018. System-level optimal yield: increased value, less risk, improved stability, and better fisheries. Can. J. Fish. Aquat. Sci. 75: 1-16.

Loreau, M., and C. de Mazancourt. 2008. Species synchrony and its drivers: neutral and nonneutral community dynamics in fluctuating environments. Am. Nat. 172: E48-E66.

Minto, C., J. Mills Flemming, G.L. Britten, B. Worm, and K. Rose. 2014. Productivity dynamics of Atlantic cod. Can. J. Fish. Aquat. Sci. 71: 203-216.

Peterman, Randall M., and B. Dorner. 2012. A widespread decrease in productivity of Sockeye Salmon (Oncorhynchus nerka) populations in western North America. Can. J. Fish. Aquat. Sci. 69: 1255-1260.

Punt, A.E., D.S. Butterworth, C.L. de Moor, J.A. A. De Oliveira, and M. Haddon. 2016. Management strategy evaluation: best practices. Fisheries 17: 303-334.

Satterthwaite, W.H., and S.M. Carlson. 2015. Weakening portfolio effect strength in a hatchery-supplemented Chinook salmon population complex. Can. J. Fish. Aquat. Sci. 72: 1860-1875.

Thibaut, L.M., and S.R. Connolly. 2013. Understanding diversity-stability relationships: towards a unified model of portfolio effects. Ecol. Lett. 16: 140-150.

Tilman, D. 1999. The ecological consequences of changes in biodiversity: a search for general principles. Ecol. 80: $1455-1474$. 